\title{
Aberrant Seizure-Induced Neurogenesis in Experimental Temporal Lobe Epilepsy
}

\author{
Jack M. Parent, MD, ${ }^{1}$ Robert C. Elliott, PhD, ${ }^{2}$ Samuel J. Pleasure, MD, PhD, ${ }^{3}$ Nicholas M. Barbaro, MD, ${ }^{4}$
} and Daniel H. Lowenstein, $\mathrm{MD}^{3}$

\begin{abstract}
Neurogenesis in the hippocampal dentate gyrus persists throughout life and is increased by seizures. The dentate granule cell (DGC) layer is often abnormal in human and experimental temporal lobe epilepsy, with dispersion of the layer and the appearance of ectopic granule neurons in the hilus. We tested the hypothesis that these abnormalities result from aberrant DGC neurogenesis after seizure-induced injury. Bromodeoxyuridine labeling, in situ hybridization, and immunohistochemistry were used to identify proliferating progenitors and mature DGCs in the adult rat pilocarpine temporal lobe epilepsy model. We also examined dentate gyri from epileptic human hippocampal surgical specimens. Prox-1 immunohistochemistry and pulse-chase bromodeoxyuridine labeling showed that progenitors migrate aberrantly to the hilus and molecular layer after prolonged seizures and differentiate into ectopic DGCs in rat. Neuroblast marker expression indicated the delayed appearance of chainlike progenitor cell formations extending into the hilus and molecular layer, suggesting that seizures alter migratory behavior of DGC precursors. Ectopic putative DGCs also were found in the hilus and molecular layer of epileptic human dentate gyrus. These findings indicate that seizure-induced abnormalities of neuroblast migration lead to abnormal integration of newborn DGCs in the epileptic adult hippocampus, and implicate aberrant neurogenesis in the development or progression of recurrent seizures.
\end{abstract}

Ann Neurol 2006;59:81-91

The pathophysiological basis of the mesial temporal lobe epilepsy (mTLE) syndrome, a particularly intractable and common form of human epilepsy, is unknown. Prior studies suggest that alterations involving the hippocampal formation, such as neuronal loss and astrogliosis in the pyramidal cell layer and dentate hilus, are pathophysiologically important (see Dalby and Mody $^{1}$ for review). Despite being relatively preserved in mTLE, the dentate granule cell (DGC) layer often shows abnormalities such as reorganization of the DGC mossy fiber axons (mossy fiber sprouting), dispersion of the cell layer, and the appearance of ectopic granule-like neurons in the hilus and molecular layer. ${ }^{2,3}$

The importance of these pathological abnormalities in the epileptic human hippocampal formation remains undetermined, but similar pathology is recapitulated in adult rodent models of mTLE. For the dentate gyrus, the combination of mossy fiber sprouting, hilar cell loss, and DGC dispersion occurs in the pilocarpine model of mTLE. ${ }^{4}$ Several groups also have found hilar ectopic granule-like neurons after pilocarpine- or kainic acid-induced status epilepticus (SE) ${ }^{5-8}$ Accumulating evidence suggests that the ectopic DGCs are abnormally integrated and hyperexcitable, and thus may contribute to seizure generation or propagation. ${ }^{7-9}$

DGC and subventricular zone (SVZ)-olfactory bulb neurogenesis persists in the adult mammalian brain and is increased by seizures. ${ }^{5,10-17}$ Pilocarpine-induced SE in adult rats stimulates SVZ neurogenesis and alters neuroblast migration to the olfactory bulb. ${ }^{17}$ Neurogenesis in the adult rodent dentate gyrus also increases in experimental mTLE. ${ }^{5,16,18-20}$ After pilocarpineinduced SE, both newborn and mature DGCs contribute to aberrant mossy fiber reorganization, ${ }^{5,21}$ and cells expressing neuroblast markers and granule cell morphology appear in the dentate hilus and molecular layer several weeks after pilocarpine treatment. ${ }^{5}$ These putative DGC progenitors resemble the "ectopic" granule-like neurons identified in surgical specimens from humans with $\mathrm{mTLE}^{2}$ and are found up to 21 months after chemoconvulsant-induced SE. ${ }^{7}$ However, the origin and specific phenotypes of newly generated neurons in the dentate hilus and molecular layer of epileptic animals, as well as the mechanisms leading to
From the ${ }^{1}$ Department of Neurology, University of Michigan Medical Center, Ann Arbor, MI; ${ }^{2}$ National Institute of Neurological Disorders and Stroke, National Institutes of Health, Bethesda, MD; and Departments of ${ }^{3}$ Neurology and ${ }^{4}$ Neurosurgery, University of California, San Francisco, San Francisco, CA.

Received Jul 6, 2005, and in revised form Sep 6. Accepted for publication Sep 12, 2005.
Published online Oct 31, 2005 in Wiley InterScience (www.interscience.wiley.com). DOI: 10.1002/ana.20699

Address correspondence to Dr Parent, Department of Neurology, University of Michigan Medical Center, 4412 Kresge III, 200 Zina Pitcher Place, Ann Arbor, MI 48109-0585.

E-mail: parent@umich.edu 
aberrant neurogenesis after seizure-induced injury, are unknown.

We sought to determine whether hilar ectopic granule-like neurons arise from neural precursors in the dentate subgranular zone (SGZ), and if so, how DGC precursors are redirected to aberrant locations after SE. We found that many proliferating cells in the dentate SGZ migrate in chains to the hilus and molecular layer and express a DGC phenotype within 2 weeks after seizure-induced injury. Similar ectopically located granule-like neurons appeared in the hilus and molecular layer of epileptic human dentate gyrus. Together with data from prior studies of hilar-ectopic DGCs, ${ }^{7-9}$ these findings suggest that aberrant adult neurogenesis contributes to network abnormalities and seizure phenotype in the epileptic hippocampal formation.

\section{Materials and Methods}

\section{Pilocarpine-Induced Status Epilepticus}

Young adult male Sprague-Dawley rats $(200-240 \mathrm{gm})$ were pretreated with injections of atropine methylbromide $(5 \mathrm{mg} / \mathrm{kg}$ intraperitoneally [IP]; Sigma, St. Louis, MO), and 15 minutes later were given pilocarpine hydrochloride $(340 \mathrm{mg} / \mathrm{kg}$ IP; Sigma) to induce SE. If seizure activity was not initiated within 1 hour after the initial pilocarpine hydrochloride dose, an additional dose of $170 \mathrm{mg} / \mathrm{kg}$ was given. Seizures were monitored behaviorally, and then terminated with diazepam $(10 \mathrm{mg} / \mathrm{kg}$ IP; Elkins-Sinn, Cherry Hill, NJ) after 2 hours of focal convulsive SE (head and forelimb clonus); additional doses of $5 \mathrm{mg} / \mathrm{kg}$ diazepam were given at 45 to 60-minute intervals if more subtle convulsive seizure activity persisted. Control rats were treated identically except that $0.9 \%$ sodium chloride solution was substituted for pilocarpine. All procedures were approved by research oversight committees for the use and care of animals at the University of California, San Francisco and the University of Michigan Medical Centers.

\section{Bromodeoxyuridine Labeling and Rat Brain \\ Tissue Processing}

Pulse-chase bromodeoxyuridine (BrdU) labeling was used to identify proliferating (S-phase) cells and follow their disposition over time. $\mathrm{BrdU}(50 \mathrm{mg} / \mathrm{kg}$; Roche, Indianapolis, IN) was administered twice, 6 hours apart, on day 7 after saline or pilocarpine treatment $(n=3-6$ per time point for each group), and animals were perfused 2, 7, or 28 days later (9, 14, or 35 days after SE). Rats received an overdose of pentobarbital sodium (Abbott Laboratories, North Chicago, IL) and were transcardially perfused with phosphate-buffered saline followed by $4 \%$ paraformaldehyde ( $\mathrm{pH} 7.4 ; 360 \mathrm{ml})$. Additional animals ( $\mathrm{n}=4-5$ per group) were perfused 21 days after pilocarpine or saline treatment. After postfixation in situ overnight, brains were removed, washed once in phosphate-buffered saline, cryoprotected with $30 \%$ sucrose in phosphate-buffered saline, and frozen in powdered dry ice. Brains were sectioned coronally at $40 \mu \mathrm{m}$ thickness through the entire rostrocaudal extent of the hippocampal formation.

\section{Human Hippocampal Tissue Processing}

Hippocampal tissue specimens from five patients (three men, two women; age range, 32-54 years) were examined. All patients were diagnosed with pharmacoresistent mTLE by ictal and interictal electroencephalographic recordings with video monitoring, brain magnetic resonance imaging, and positron emission tomography scans. Anterior temporal epilepsy surgeries were performed at the University of California, San Francisco Medical Center between February and November 1999. Hippocampi were resected en bloc, and small portions of approximately $0.5 \mathrm{~cm}$ in length were sectioned coronally into 1 to $2 \mathrm{~mm}$ blocks, immersion fixed in $4 \%$ paraformaldehyde (PFA), and cryoprotected and frozen as described earlier for rat brain tissue. Hippocampal tissue was sectioned coronally at $40 \mu \mathrm{m}$ thickness with a cryostat into tris(hydroxymethyl)aminomethane (Tris) buffer (0.1M). Neuropathologists examined representative specimens of lateral temporal cortex, white matter, amygdala, and hippocampus. A diagnosis of mesial temporal sclerosis without dual pathology was made in all five cases. Paraffin-embedded hippocampal blocks from five age-matched subjects without neurological disease were used as control samples. Hippocampi were sectioned on a microtome at 20 to $30 \mu \mathrm{m}$ thickness and airdried onto gelatin-coated slides (Superfrost-plus; Fischer Scientific, Fair Lawn, NJ). Acquisition of human tissue was approved by the Committee on Human Research of the University of California, San Francisco.

\section{In Situ Hybridization and Immunohistochemistry}

Nonradioactive in situ hybridization to detect Prox-1 and Mash1 mRNA was performed using digoxigenin-labeled antisense riboprobes, as described previously. ${ }^{22,23}$ Diaminobenzidine peroxidase immunohistochemistry was performed on free-floating tissue sections or slides according to published protocols. ${ }^{5,17}$ For BrdU immunostaining, DNA was denatured by incubating tissue sections in $2 \mathrm{~N} \mathrm{HCl}$ for 30 minutes at $37^{\circ} \mathrm{C}$, followed by a 10 -minute wash in $0.1 \mathrm{M}$ borate solution, $\mathrm{pH}$ 8.5. Tissue was incubated overnight in primary antibody at $4^{\circ} \mathrm{C}$; antibody dilutions used were: $1: 1,000$ for BrdU (mouse monoclonal; Boehringer Mannheim, Indianapolis, IN), 1:5,000 for doublecortin (DCx; rabbit polyclonal; a gift of C. Walsh, Harvard University, Cambridge, MA); and 1:5,000 to $1: 10,000$ for Prox-1 (rabbit polyclonal). ${ }^{24}$ Single- and double-label immunofluorescence histochemistry was done according to previously described methods. ${ }^{5,17}$ Primary antibody dilutions used were: 1:200 for BrdU (rat monoclonal; Accurate Chemical, Westbury, NY); 1:500 for polysialylated neural cell adhesion molecule (PSA-NCAM; mouse IgM 5A5 clone; Developmental Studies Hybridoma Bank, University of Iowa, Iowa City, IA); 1:10,000 for TUC-4 (also known as CRMP-4; rabbit polyclonal; a gift of S. Hockfield, Yale University, New Haven, CT); 1:1,000 for neuronal nuclear protein (NeuN; mouse monoclonal; Chemicon, Temecula, CA); 1:500 for microtubule-associated protein 2 (MAP2; mouse monoclonal; Sigma), and 1:200 for glial fibrillary acidic protein (GFAP; rat monoclonal; a gift of V. Lee, University of Pennsylvania, Philadelphia, PA). Secondary antibodies for immunofluorescence were goat antirat IgG conjugated to fluorescein isothiocyanate or Texas Red, goat anti-mouse IgG conjugated to fluorescein isothio- 
cyanate or Texas Red, goat anti-rabbit IgG conjugated to fluorescein isothiocyanate or Texas Red, and goat antimouse IgM conjugated to Texas Red (all species crossadsorbed and from Jackson Immunoresearch Laboratories, West Grove, PA) at 1:400 dilutions. Tissue was incubated in secondary antibody overnight at $4^{\circ} \mathrm{C}$. For double-label immunofluorescence using rat anti-BrdU and a second primary antibody, free-floating sections were incubated in the second primary antibody for 24 hours at $4^{\circ} \mathrm{C}$, washed with Trisbuffered saline $(0.15 \mathrm{M} \mathrm{NaCl}, 0.1 \mathrm{M}$ Tris- $\mathrm{HCl}, \mathrm{pH} 7.6)$ for 45 minutes, postfixed in 4\% PFA for 20 minutes, rinsed 3 times with Tris-buffered saline, and denatured with $2 \mathrm{~N} \mathrm{HCl}$, as described earlier. After Tris-buffered saline washes and blocking, sections were incubated in rat anti-BrdU antibody and the second primary antibody for another 24 hours at $4^{\circ} \mathrm{C}$.

\section{Microscopy, Cell Counts, and Statistical Analyses}

For double-label immunofluorescence, images were obtained using a BioRad MRC 1024 (BioRad, Hercules, CA) or Zeiss LSM 510 (Zeiss, Oberkochen, Germany) confocal laser microscope as $z$-series stacks (1.0 $\mu \mathrm{m}$ optical thickness), visualized with National Institutes of Health Image version 1.61 software, and transferred to Adobe Photoshop for color merging (Adobe Systems, Mountain View, CA). Light microscopic and single-labeled epifluorescence microscopic images were acquired under a $20 \times$ objective using a Leica DMIRB (Leica, Heidelberg, Germany) inverted microscope and SPOT-RT (SPOT Diagnostic Instruments, Sterling Heights, MI) digital camera. BrdU- and Prox-1-immunoreactive cells were counted in digitized images using National Institutes of Health image 1.61 software with the examiner blinded to experimental condition. The region of interest was selected manually using a wand and drawing tablet and included the hilus between the two blades of the DGC layer excluding two cell body widths interior to the DGC layer (ie, excluding the SGZ). Counts were made from 4 to 5 rats/group using 5 to 6 sections (10-12 dentate gyri) spanning the entire rostrocaudal extent of the hippocampus. Statistical comparisons between groups for counts of $\mathrm{BrdU}$ (each time point compared separately due to the pulse-chase protocol) were made using two-tailed Student's $t$ test, and for Prox-1 with analysis of variance and Fisher's protected least-squares difference post hoc test. Analyses were performed with StatView software (Abacus Concepts, Berkeley, $\mathrm{CA})$; the significance level was set at $p<0.05$.

\section{Results}

Status Epilepticus Induces the Delayed Appearance of Hilar Ectopic Dentate Granule Cells

Hilar ectopic granule-like neurons are found in the adult rodent dentate gyrus after chemoconvulsantinduced SE. ${ }^{5,7,8}$ They are found within 1 month and persist for up to 21 months after SE, but the timing of their initial appearance after seizures is unknown. To determine the time course of hilar granule-like neuron induction after pilocarpine-induced SE, we first examined the expression of the divergent homeobox gene Prox-1 as a marker for DGCs. Prox-1 is expressed in postmitotic DGCs and is specific for this cell type in the adult rat dentate gyrus. ${ }^{22-24}$ In situ hybridization for Prox-1 mRNA and immunohistochemistry with a Prox-1-specific antibody ${ }^{24}$ showed the expected pattern of expression restricted to the DGC layer in saline-treated control adult rats (Figs 1A, E). This pattern was essentially unchanged at 7 days after pilocarpine-induced SE (see Figs 1B, F). Within 2 weeks after pilocarpine treatment, however, Prox-1-expressing cells appeared in the hilus and molecular layer (see Figs 1C, G), and hilar Prox-1 expression persisted for at least 5 weeks after SE (see Figs 1D, H). We quantified the numbers of Prox-1-immunoreactive cells in the hilus of saline or pilocarpine-treated animals and found significant increases at both 14 and 35 days after SE (Fig 2H). These data suggest that prolonged seizures induce a delayed accumulation of DGCs in the hilus.

\section{Hilar Ectopic Dentate Granule Cells Are Newly Generated Neurons That Arise from Dentate Subgranular Zone Precursors}

Because Prox-1 is expressed by differentiated and not recently postmitotic DGCs, ${ }^{22}$ the delayed accumulation of hilar Prox-1-expressing cells raises the possibility that SGZ neuroblasts migrate to the hilus after SE, and then differentiate into DGCs. ${ }^{5}$ Alternatively, the cells could be generated in situ in the hilus, or mature DGCs could move to the hilus after seizure-induced injury. To test the first possibility, that the hilar cells are newly generated after seizures and migrate from the SGZ to the hilus, we performed a series of pulse-chase BrdU-labeling experiments after seizure induction to identify proliferating progenitors and follow their dispersal over time. BrdU was administered to adult rats 1 week after treatment with saline or pilocarpine, and animals survived for an additional 2, 7, or 28 days. In control rats, BrdU labeling was largely restricted to the SGZ at day 9 after saline treatment (2 days after BrdU administration; see Fig 2A, arrows); over time they migrated a short distance into the hilar aspect of the granule cell layer (see Figs 2C, E, G), as expected., ${ }^{5}$ At day 9 after SE, most BrdU-immunoreactive cells remained in the SGZ (see Fig 2B, arrows), although increased numbers were already present in the hilus (see Fig 2G); however, these cells tended to be close to the SGZ. In contrast, within 14 days after seizures (and 7 days after BrdU administration), many BrdU-labeled cells appeared throughout the hilus and in the molecular layer (see Figs 2D, G); the numbers were significantly increased compared with control cells at that time point, and many hilar BrdU-positive cells persisted for at least 35 days after SE (see Figs 2F, G). The degree of hilar BrdU labeling declined between 14 and 35 days after SE (see Fig 2G), perhaps due to cell death or dilution of BrdU content below detectible lev- 


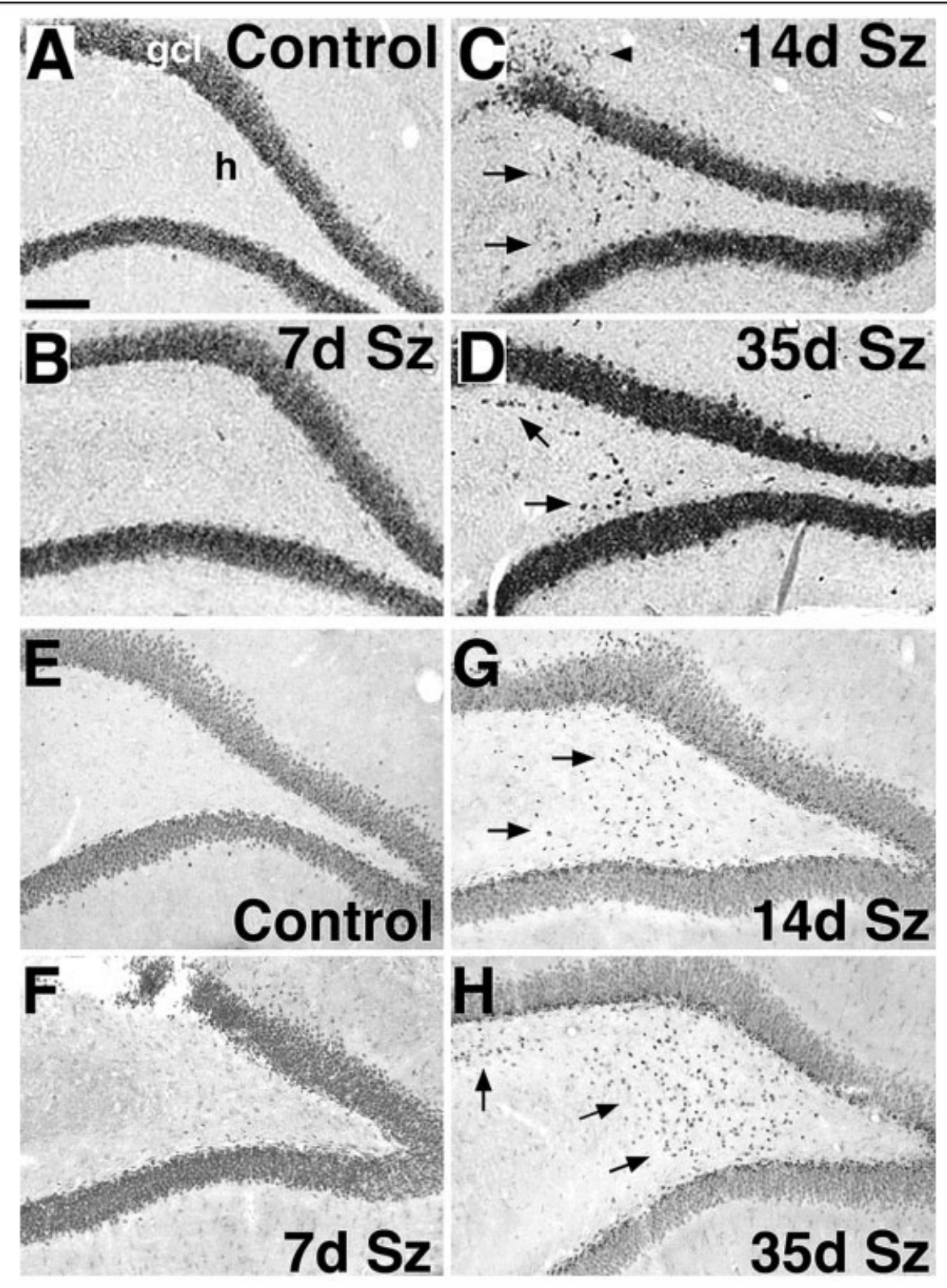

Fig 1. Prox-1-expressing cells accumulate in the dentate hilus and molecular layer after status epilepticus (SE). (A-D) Nonradioactive in situ hybridization using a Prox-1 riboprobe on coronal sections through the dentate gyrus of a saline-treated control animal (A) and animals 7 (B), $14(C)$, or 35 (D) days after pilocarpine-induced SE. (E-H) Immunohistochemistry for Prox-1 in a control (E) and three different pilocarpine-treated rats at the same time points as in A through D. Prox-1 expression is restricted to cells in the dentate granule cell layer $(\mathrm{gcl})$ of control animals $(A, E)$ and animals 7 days after SE $(B, F)$. By 14 days after SE, Prox-1 messenger RNA $(C)$ and protein $(G)$ expression markedly increases in the dentate hilus ( $h$; arrows) and molecular layer (arrowhead in $C$ ), and persists up to 5 weeks after SE (arrows in D, H). The arrowhead in $C$ shows Prox-1 expressing cells in the molecular layer 2 weeks after SE. Scale bar $=100 \mu \mathrm{m}$.

els by multiple cell divisions. The persistence of large numbers of Prox-1-immunoreactive cells in the hilus at 35 days (see Figs $1 \mathrm{H}$ and $2 \mathrm{H}$ ), however, suggests that many of the newly generated cells survived and differentiated into DGCs.

The delayed appearance of both BrdU-labeled and Prox-1-expressing cells in the hilus after pilocarpine treatment suggests that the hilar Prox-1-immunoreac- tive cells are newly generated DGCs. To test this idea, we performed immunofluorescence double labeling for BrdU and Prox-1 at 35 days after SE (28 days after BrdU administration). Confocal microscopic analysis indicated that the majority of hilar BrdU-positive cells expressed Prox-1 (Fig 3A, arrows), as did those in the granule cell layer (see Fig 3A, arrowhead). Hilar BrdUlabeled cells at this time point after seizures also ex- 


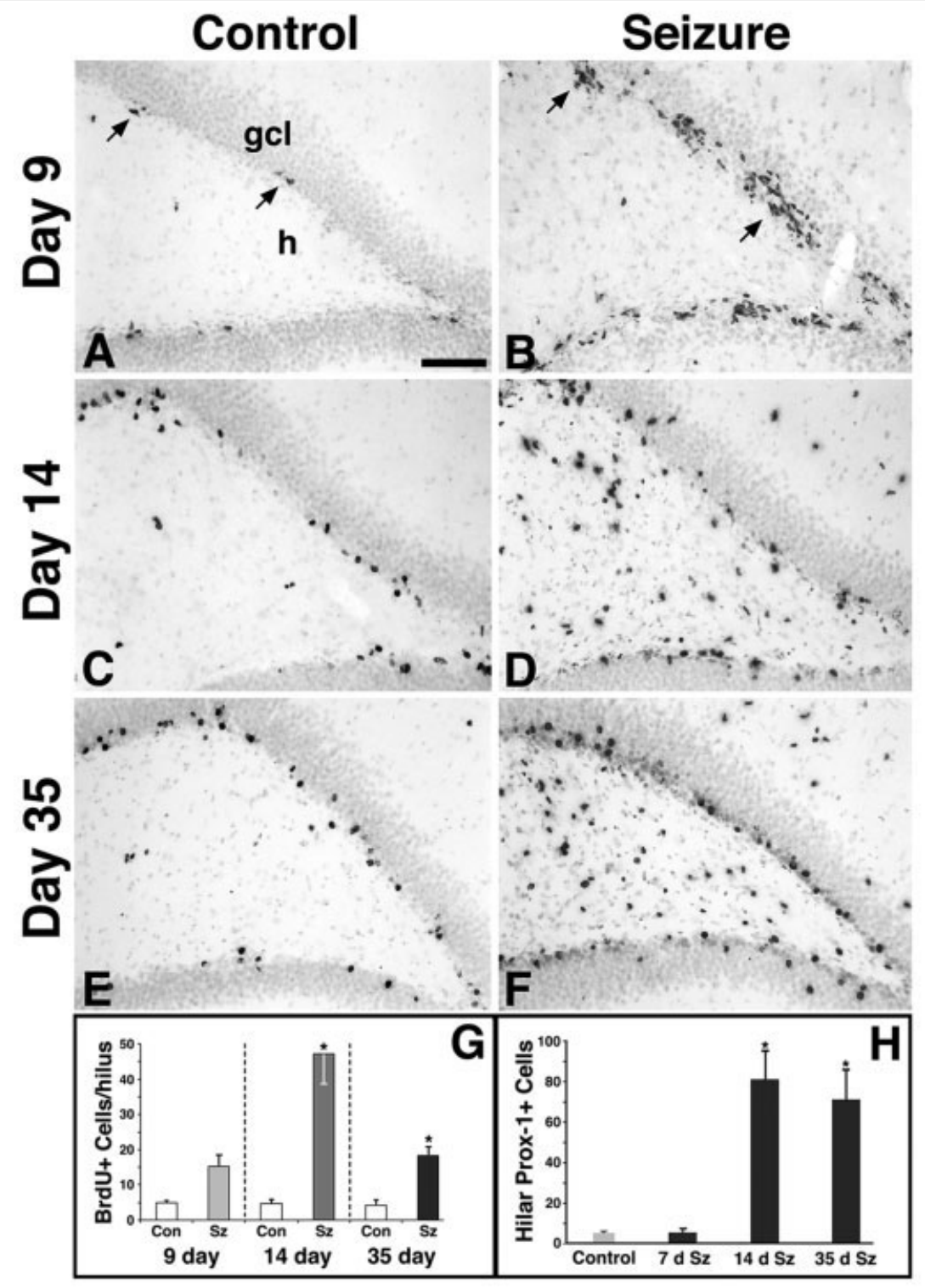

Fig 2. Pulse-chase bromodeoxyuridine (BrdU) labeling shows seizure-induced migration of cells from the dentate subgranular zone $(S G Z)$ to hilus. $(A-F)$ Coronal sections through the dentate gyrus after saline $(A, C, E)$ or pilocarpine treatment $(B, D, F)$. Brd $U$ was administered on day 7 after treatment, and animals were killed $2(A, B), 7(C, D)$, or 28 (E, F) days later. Note the relative restriction of $B r d U$-immunoreactive cells in the $S G Z$ (arrows in $A, B$ ) or granule cell layer of control animals $(A, C, E)$ and a rat 9 days after status epilepticus (SE) and 2 days after BrdU administration (B), compared with the extensive hilar BrdU labeling at $14(D)$ and 35 (F) days after seizures (7 and 28 days after BrdU administration). Quantification of hilar BrdU- (G) and Prox1-immunoreactive (H) cells after SE shows significantly increased BrdU labeling in the hilus at 14 and 35 days after pilocarpine treatment compared with control animals ( $\left.{ }^{*} \mathrm{p}<0.005\right)$; a trend toward increased Brd ${ }^{+}$cells was seen for the 9-day seizure group ( $\mathrm{p}=0.055)$. Hilar Prox-1 expression was significantly increased in the $14-(n=5)$ and 35 -day $(n=4)$ seizure groups compared with control animals $(n=7)$ and the 7 -day seizure group $\left(n=4 ;{ }^{*} \mathrm{p}<.001\right)$. h, hilus; gcl, granule cell layer. Scale bar $=100 \mu \mathrm{m}$.

pressed the neuron-specific markers NeuN and MAP2 (see Figs 3B, C). At 14 days after pilocarpine treatment, when the BrdU-positive cells would still be expected to be immature, these newly generated cells expressed immature neuronal markers such as PSANCAM and TUC-4 (see Fig 3D; further data not shown). These data indicate that neuronal progenitors migrate aberrantly into the hilus after SE, where they differentiate into hilar ectopic DGCs.

Status Epilepticus Induces Aberrant Neuroblast Chain Migration from the Dentate Subgranular Zone

The Prox-1 expression and pulse-chase BrdU-labeling data presented earlier show that hilar ectopic DGCs 

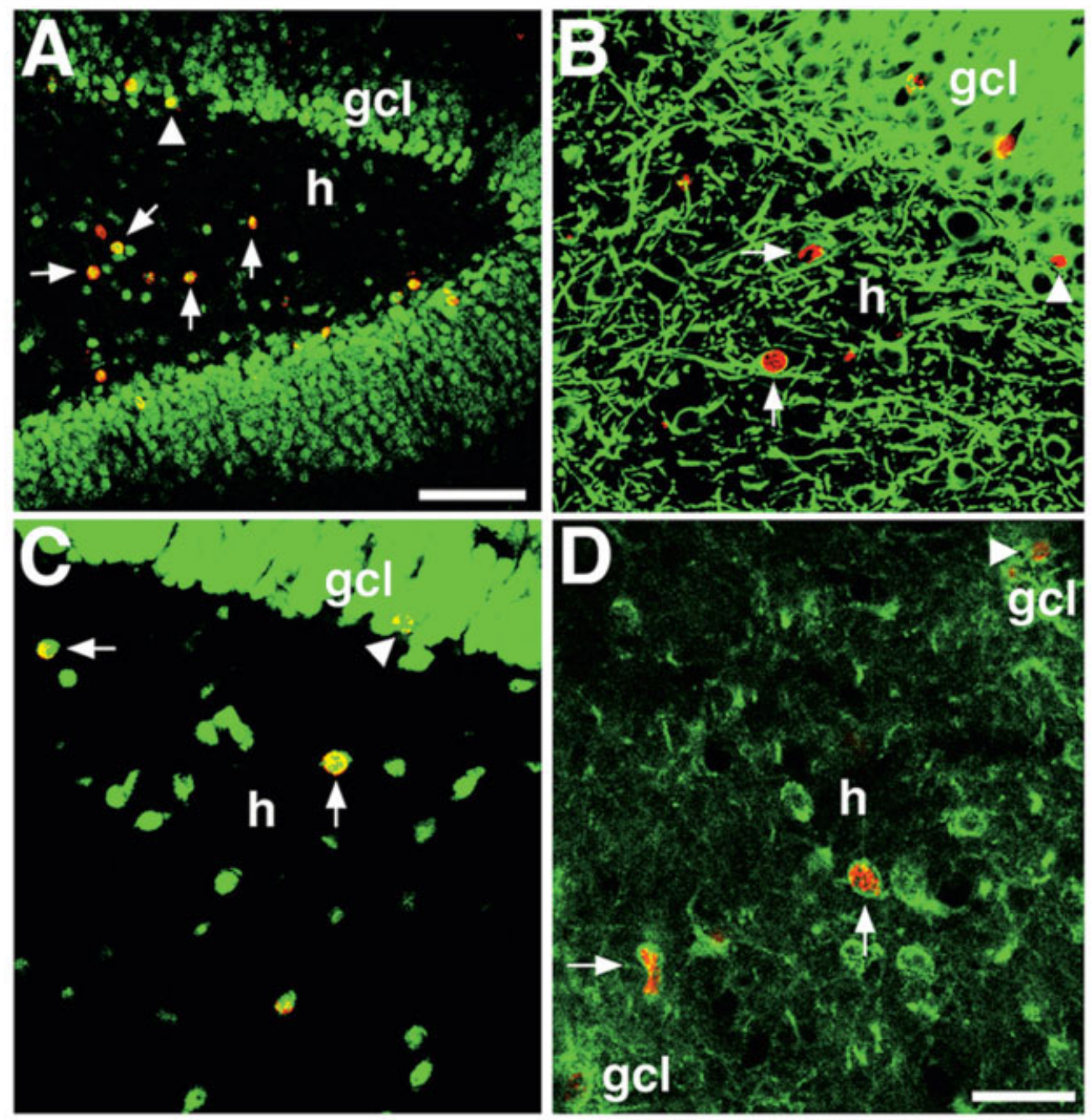

Fig 3. Newly generated hilar cells express markers specific for neurons and dentate granule cells (DGCs). Confocal images of fluorescence immunostaining $35(A-C)$ or 14 (D) days after status epilepticus (SE) show double labeling for bromodeoxyuridine (BrdU) (red in all panels) and Prox-1 (A), microtubule-associated protein 2 (B), neuronal nuclear protein (C), or polysialylated neural cell adhesion molecule (D). Note double-labeled cells (either yellow or red nucleus surrounded by green cytoplasm) in the hilus (h; arrows) and inner DGC layer (gcl; arrowheads). BrdU always was given 7 days after SE. Scale bar $=40 \mu m(A) ; 25 \mu m(B-D)$.

arise from the dentate SGZ and accumulate in the hilus over several weeks after SE. Migrating neuroblasts should therefore be evident in the dentate SGZ and hilus as the cells accumulate. Neuroblasts in the adult rodent forebrain SVZ and dentate SGZ express specific markers, such as DCx, TUC-4, and PSA-NCAM, as they migrate and differentiate. ${ }^{5,17,25-30}$ The expression patterns of these markers previously allowed us to identify ectopic neuroblast migration in the forebrain SVZolfactory bulb pathway after seizure- or stroke-induced injury. ${ }^{17,31}$ We therefore performed immunohistochemistry for DCx, TUC-4, and PSA-NCAM to determine whether neuroblasts migrate aberrantly in the epileptic dentate gyrus after pilocarpine treatment.

In the intact adult rat dentate gyrus, DCx, TUC-4, and PSA-NCAM expression was confined to cells located in the dentate SGZ and inner DGC layer (Fig
4A; further data not shown), the region of newly generated neurons, as described previously. ${ }^{5,25}$ Immunoreactivity was present in cell bodies, axons, and dendrites of the immature DGCs (see Figs 4A, C). Expression of neuroblast markers increased in the SGZ and, in some instances, in the molecular layer of the superior blade, but not in the hilus, at 7 days after pilocarpine treatment (data not shown). By 14 days after SE, DCx, TUC-4, and PSA-NCAM immunoreactivity increased markedly in the dentate SGZ, hilus, and molecular layer (see Figs 4B, D). Many chainlike formations of DCx- and TUC-4-immunoreactive neuroblasts extended from the SGZ to the hilus at 14 and 21 days after pilocarpine treatment (see Figs 4D, E, G); these were largely gone by 35 days (see Fig 4F). These structures resembled the chains formed by forebrain SVZ neuroblasts that migrate tangentially to the olfactory 


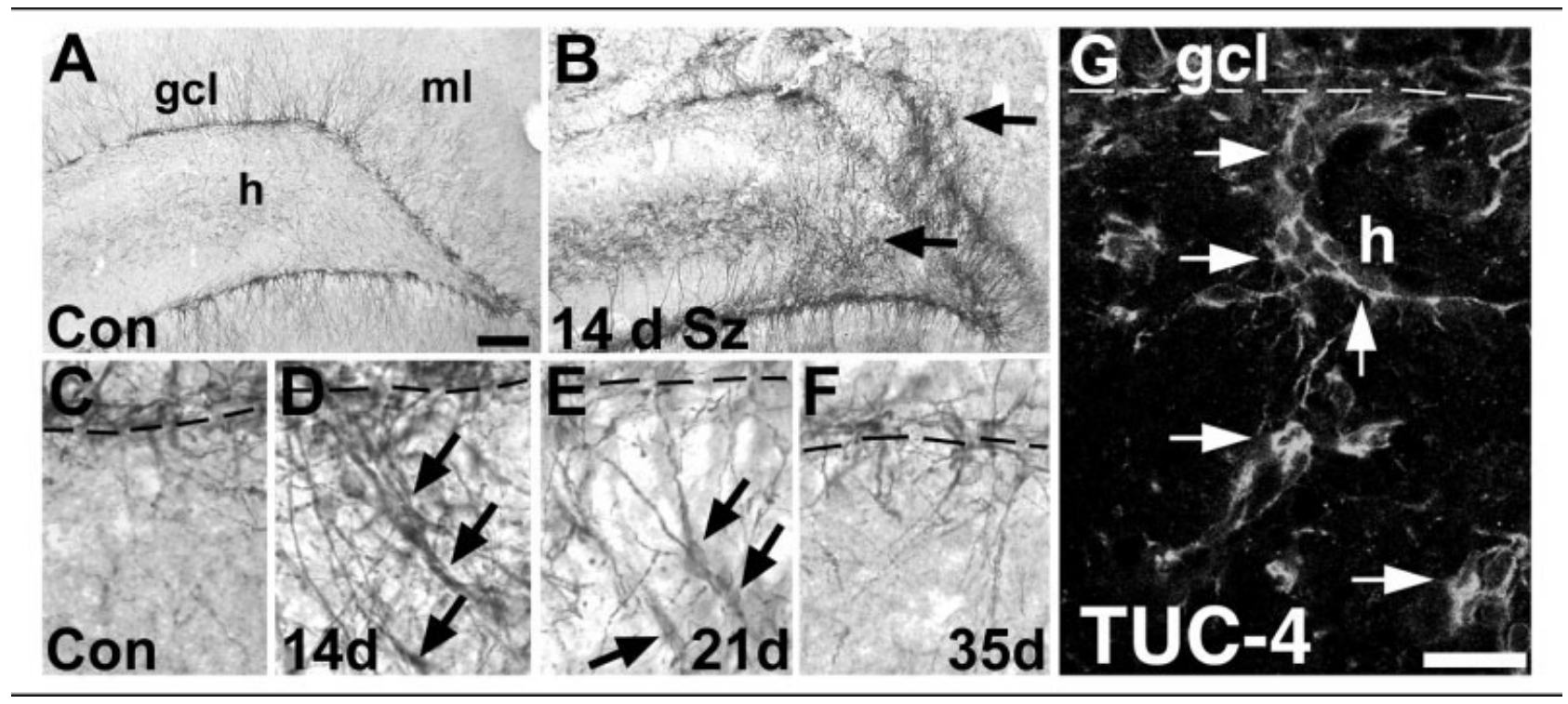

Fig 4. Seizure-induced neuroblast chain migration from the dentate subgranular zone (SGZ) to hilus. (A-F) Coronal sections through the adult rat dentate gyrus immunostained for doublecortin $(D C x)$. $(A, B)$ Lower magnification images show the marked increase in DCx immunoreactivity 14 days after status epilepticus (SE) (B) compared with control animal (A). In addition to the $S G Z$ and inner dentate granule cell layer (gcl), note that DCx expression is markedly increased in the hilus ( $h$ ) and molecular layer ( $m l$; arrows in B; compare with $A)$. (C-F) DCx-immunoreactive cells are largely confined to the inner gcl in a control animal (C); at 14 (D) and 21 (E) days after SE, however, chains of DCx-positive cells extend from the inner gcl to the hilus (arrows). The pattern of DCx expression resembles that of control animals by 35 days after pilocarpine treatment $(F)$. ( $G$ ) Confocal optical image of TUC-4 immunohistochemistry showing labeling of a chain of neuroblasts extending from the inner gcl to the hilus, as well as clusters of TUC-4-positive cells deep in the hilus (arrows). (C-G) Dashed lines denote the gcl/hilar border. Scale bars $=100 \mu m$ $(A, B) ; 40 \mu m(C-F$, bar appears in $H)$; and $30 \mu m(H)$.

bulb in intact adult rodents ${ }^{27,32,33}$; such chainlike formations were never seen in the dentate gyrus of salinetreated control animals (see Fig 4C).

DCx-positive cells in the hilus 21 days after SE had the characteristic morphology of DGCs. The cells displayed large, "apical" dendrites and mossy fiber-like axons, and they appeared highly disorganized, with cell bodies oriented in different planes and processes projecting in varying directions. We performed immunofluorescence double labeling for the astrocytic protein GFAP and the neuoroblast markers DCx, TUC-4, or PSA-NCAM to confirm the neuronal specificity of the latter proteins. Neither control nor pilocarpine-treated rats 14 days after SE showed cellular colocalization of astrocytic and neuroblast markers, although astrocytes and neuroblasts were often closely apposed, as described previously for the intact adult rat DGC layer. ${ }^{34}$

Similar patterns of ectopic DGC progenitors, proliferating cells, and chains of neuroblasts also appeared in the dentate molecular layer of the superior blade in some pilocarpine-treated rats. Ectopic molecular layer neurogenesis usually occurred in animals that displayed significant injury after SE, typically involving the superolateral blade of the DGC layer. DGC progenitors express the basic helix-loop-helix protein Mash-1 during early development and in the adult. ${ }^{22,23}$ One week after SE, Mash-1-expressing cells were sometimes found in the dentate molecular layer in addition to the SGZ (Fig 5A). BrdU-immunoreactive cells appeared in the molecular layer 1 to 2 weeks after seizures in animals with extensive injury (see Figs 2D, 2F, and 5B), and molecular layer BrdU labeling coincided with expression of Prox-1, DCx, TUC-4, and PSA-NCAM similar to that found in the hilus (see Figs 1C, 4B, 5C, and 5D). As in the hilus, chainlike configurations of neuroblasts were often found (see Fig 5D). Thus, pilocarpine-induced SE altered the migratory behavior of dentate SGZ progenitors, leading to the ectopic appearance of newly generated DGCs in both the dentate hilus and molecular layer.

\section{Dentate Gyri of Humans with Mesial Temporal Lobe Epilepsy Contain Ectopic Granule-like Neurons} The finding of hilar- and molecular-layer ectopic DGCs in the pilocarpine model of mTLE led us to explore in more detail the DGC layer of patients with mTLE. We first immunostained surgically resected hippocampi from patients with pathologically confirmed mesial temporal sclerosis for the neuronal marker NeuN. As Houser ${ }^{2}$ first described, dentate gyri from epileptic patients showed marked DGC layer dispersion compared with autopsy control cases (Figs 6A, B). In the epileptic but not control tissue, many neurons with granule cell morphology appeared in the 


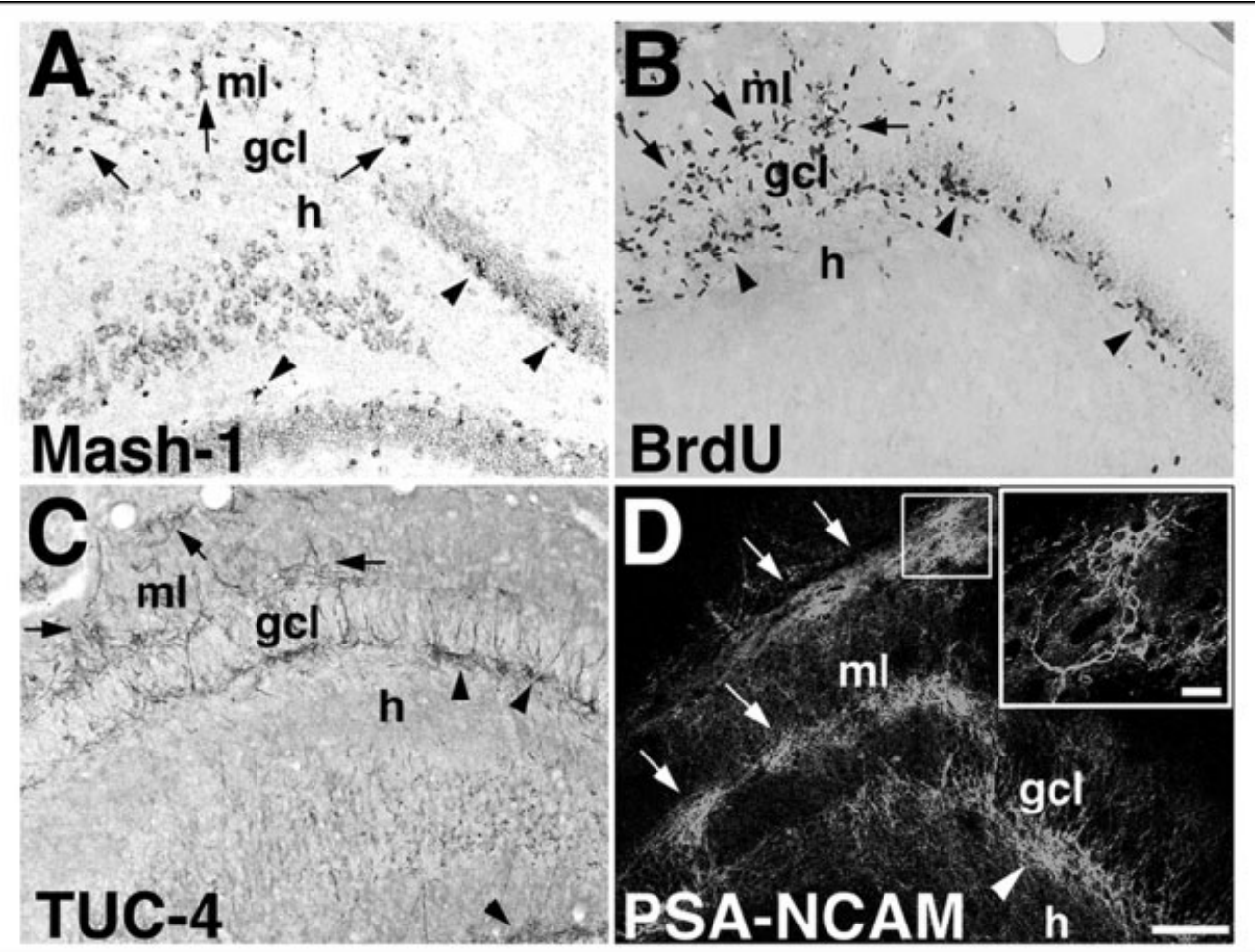

Fig 5. Molecular layer neurogenesis after prolonged seizures. (A) In situ hybridization for Mash-1, expressed by dentate granule cell $(D G C)$ progenitors, shows labeled cells in the molecular layer $(\mathrm{ml})$ of the superior blade (arrows), as well as in the subgranular zone, hilus (b), and inner granule cell layer (gcl; arrowheads) 7 days after pilocarpine treatment. (B-D) The pattern of $m l$ immunostaining (arrows) for bromodeoxyuridine (BrdU) (B) and TUC-4 (C) at 9 days, and polysialylated neural cell adhesion molecule (PSA-NCAM) (D) at 14 days after status epilepticus (SE), overlaps with that of Mash-1 at the earlier time point. Note that immature neurons expressing PSA-NCAM extend all the way to the hippocampal sulcus (arrows in D), and some assume a chainlike configuration (inset). BrdU was given 7 days after SE. Scale bar $=75 \mu \mathrm{m}(A-C) ; 100 \mu m(D) ; 20 \mu m(D$, inset).

dentate hilus and molecular layer. To provide evidence for the granule cell phenotype of the putative ectopic cells, we examined Prox-1 expression in the epileptic tissue. Prox-1-immunoreactive cells with nuclear size consistent with DGCs appeared not only in the DGC layer, but also in the dentate hilus and molecular layer (see Fig 6D). The pattern of ectopic Prox-1 immunoreactivity in epileptic human tissue was similar to that found several weeks after seizure-induced injury in the pilocarpine mTLE model (see Figs 6C, D). These findings suggest that hilar- and molecular layer-ectopic DGCs seen in human mTLE are recapitulated by the pilocarpine epilepsy model.

\section{Discussion}

These findings indicate that prolonged seizures induce aberrant neurogenesis in the adult rat dentate gyrus. Pulse-chase BrdU labeling and immunohistochemistry for immature neuronal markers showed many newly generated neurons migrating in chains from the dentate SGZ to ectopic locations in the hilus and molecular layer after SE. The newborn hilar neurons differentiated into DGCs, as identified by specific transcription factor expression and cell morphology, even though they remained outside their normal granule cell layer locations. In the dentate gyrus of humans with mTLE, but not autopsy control cases, immunostaining for the neuron-specific marker NeuN showed DGC-like neurons located in the hilus and molecular layer. This finding is unlikely to result from postmortem effects or differential processing of autopsy versus surgical specimens because NeuN immunoreactivity was robust in both groups, and dispersion has been found in autopsy specimens of patients with mTLE. ${ }^{35}$ Remarkably similar patterns of ectopic, Prox-1-immunoreactive cells were found in epileptic rat and human dentate gyrus. These findings together provide strong evidence that aberrant neurogenesis in the epileptic hippocampal formation results in the formation of ectopic DGCs.

\section{Functional Significance of Hilar Ectopic Dentate Granule Cells}

Some work suggests that hilar ectopic DGCs in adult rodent mTLE models integrate abnormally and lead to abnormal network function. ${ }^{7,8}$ Although DGCs are occasionally found in small numbers in the hilus of intact adult rats, ${ }^{36-38}$ their occurrence is rare compared with 


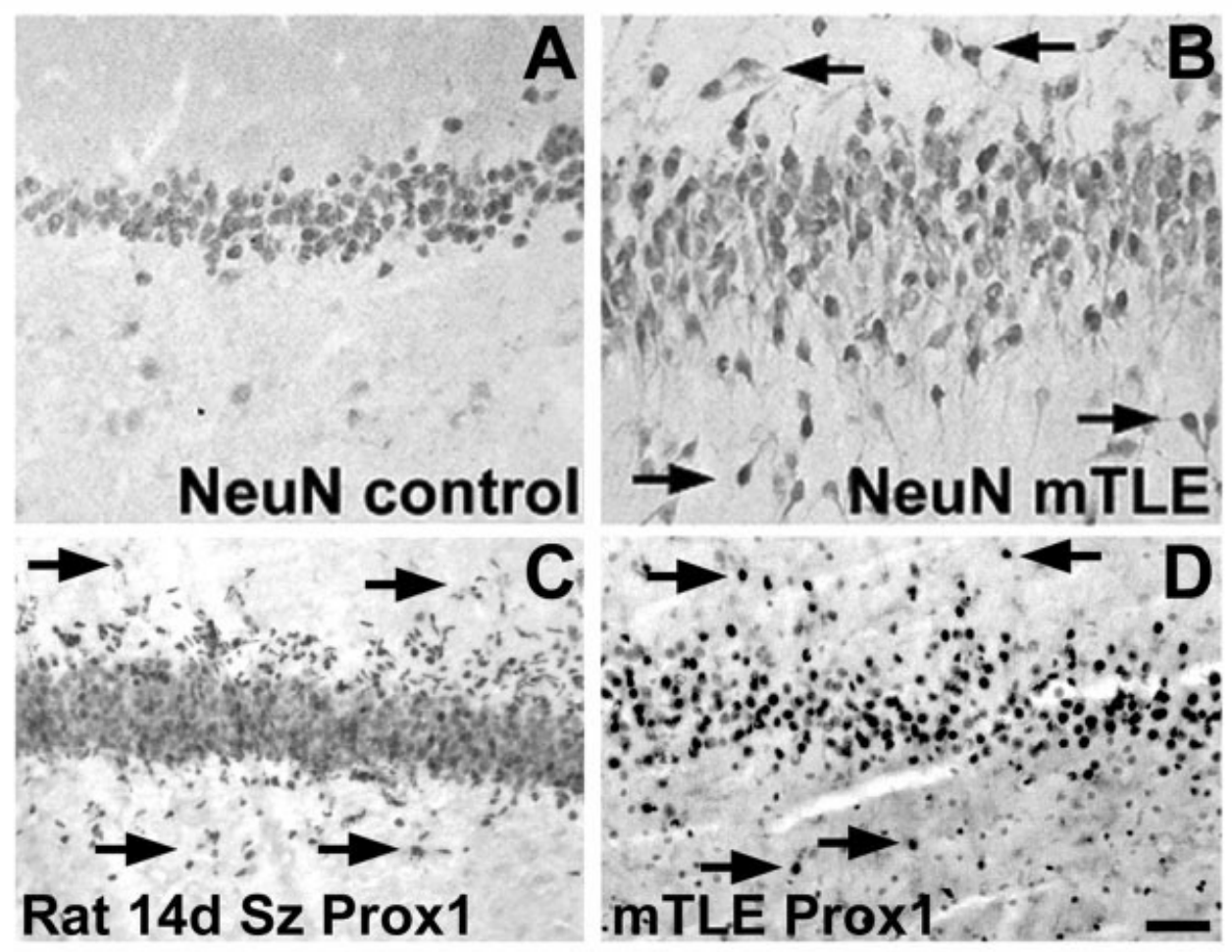

Fig 6. Human epileptic dentate gyrus shows dispersed dentate granule cell (DGC) layer and ectopic DGCs. (A, B) Coronal sections through the dentate gyrus of an autopsy control case (A) or mesial temporal lobe epilepsy (mTLE) patient (B) immunostained for neuronal nuclear protein $(N e u N)$. The DGC layer is markedly dispersed in the epileptic tissue (B), and many DGC-like cells appear in the hilus (h) and molecular layer ( $m l$; arrows in B). (C, D) Prox-1-immunostained coronal sections through the dentate gyrus of a rat 14 days after pilocarpine treatment $(C)$ and a patient with $m$ TLE (D) show similar patterns of Prox-1 expression in the DGC layer, as well as scattered Prox-1-immunoreactive cells in the hilus and molecular layer (arrows). Scale bar $=50 \mu m$ (A, $B$; bar appears in D); $100 \mu m(C, D)$.

animals experiencing prolonged seizures. Our data confirm the newborn nature and dentate SGZ progenitor cell origin of the hilar ectopic DGCs generated after SE and show that the newborn neurons persist for at least 5 weeks. Indeed, several other groups have found hilar-ectopic DGCs more than a year after pilocarpineor kainic acid-induced $\mathrm{SE},{ }^{7,8}$ suggesting that the cells are capable of prolonged integration. Scharfman and colleagues $^{7}$ examined hilar-ectopic DGC-like neurons in hippocampal slices from epileptic adult rats and found that these cells have many of the morphological and electrophysiological characteristics of DGCs. Unlike mature granule neurons in the DGC layer, however, hilar-ectopic granule cells exhibit abnormal burst firing in synchrony with CA3 pyramidal cells. ${ }^{7}$ In addition, many hilar ectopic DGCs found after seizures have bipolar dendrites that typically are present in only a small percentage of DGCs in intact adult rodents. ${ }^{6-8}$ Morphological evidence of substantially increased net excitatory synapses on the proximal dendrites of hilar ectopic DGCs ${ }^{8}$ may explain their hyperexcitability. Moreover, inhibition of neurogenesis with antimitotic agent infusion after pilocarpine-induced SE eliminates hilar-ectopic DGC formation and decreases seizure fre- quency. ${ }^{9}$ These results, together with our findings, suggest that hilar-ectopic DGCs generated from SGZ progenitors after seizure-induced injury integrate abnormally and likely contribute to network abnormalities in the epileptic hippocampal formation.

\section{Human Temporal Lobe Epilepsy and Potential Mechanisms Underlying Aberrant Neurogenesis}

Our findings of aberrant, seizure-induced DGC neurogenesis in the adult rodent have implications for the pathophysiology of human mTLE. Studies of neurogenesis in the adult human dentate gyrus and of DGC layer dispersion in tissue resected surgically to treat mTLE support this idea. DGC neurogenesis persists in adult monkeys and humans, ${ }^{39-41}$ the latter even into the eighth decade of life. ${ }^{39}$ Pathological findings in the syndrome of mTLE include dispersion of the DGC layer and the appearance of hilar ectopic granule-like neurons. ${ }^{2}$ Such findings implicate defects in persistent DGC neurogenesis as a cause of dentate gyrus structural abnormalities. ${ }^{42,43}$

Recent evidence suggests several factors involved in neural development that may underlie aberrant neurogenesis in the epileptic dentate gyrus. One candidate is 
the migration guidance cue reelin. Intact reelin signaling is necessary for normal DGC layer lamination during development, ${ }^{44}$ and Haas and colleagues ${ }^{45}$ showed that the degree of DGC layer dispersion directly correlates with decreased dentate gyrus reelin expression. Altered reelin signaling after $\mathrm{SE}$ in rat or in human mTLE may therefore lead to ectopic migration of DGC progenitors. Another factor implicated in hilarectopic DGC formation is brain-derived neurotrophic factor. Seizures result in upregulation of brain-derived neurotrophic factor in the dentate gyrus, ${ }^{46,47}$ and intrahippocamal infusion of brain-derived neurotrophic factor induces hilar-ectopic DGC formation in intact adult rats. ${ }^{48}$ Thus, seizure-induced upregulation of migration guidance cues and trophic factors may play a role in ectopic neurogenesis.

These data also suggest potential obstacles for brain reparative strategies involving endogenous or transplanted neuronal progenitors. The differentiation of SGZ progenitors that migrate to the hilus into phenotypes of hilar cells injured by seizures, such as mossy cells or GABAergic interneurons, would suggest the presence of an endogenous reparative process. Instead, dentate gyrus precursors that migrate into the hilus remain committed to a DGC phenotype despite their abnormal location. Ectopic dentate gyrus neurogenesis after seizures therefore appears to be a maladaptive, rather than compensatory, response to injury. If this is true, potential brain repair strategies using endogenous or transplanted neural precursor cells will need to overcome several obstacles to restore normal function after injury. First, altered cues may exist at sites of injury and lead to inappropriate migration of neural progenitors to undesired locations. Second, the local environment may not maintain the cues necessary to direct differentiation of endogenous or transplanted neural progenitors into the appropriate cell types. Although evidence suggests that this problem may be overcome for endogenous SVZ progenitors after striatal or hippocampal pyramidal cell ischemic injury, ${ }^{31,49,50}$ the injured dentate gyrus appears to be a less hospitable environment for neuronal replacement. A better understanding of the mechanisms regulating neural progenitor migration and differentiation in the adult will therefore be important for developing strategies to both prevent abnormal integration and promote neural repair.

This study was supported by the NIH (National Institute of Neurological Disorders and Stroke, NS02006, J.M.P., NS32062, D.H.L) and Parents Against Childhood Epilepsy, Inc. Research Grants Program.

We thank Drs S. Hockfield, V. Lee, and C. Walsh for providing antibodies, T. Wang for assistance with human tissue processing, and D. Janus for assistance with manuscript preparation.

\section{References}

1. Dalby NO, Mody I. The process of epileptogenesis: a pathophysiological approach. Curr Opin Neurol 2001;14:187-192.

2. Houser CR. Granule cell dispersion in the dentate gyrus of humans with temporal lobe epilepsy. Brain Res 1990;535: 195-204.

3. Parent JM, Lowenstein DH. Mossy fiber reorganization in the epileptic hippocampus. Curr Opin Neurol 1997;10:103-109.

4. Mello LE, Cavalheiro EA, Tan AM, et al. Circuit mechanisms of seizures in the pilocarpine model of chronic epilepsy: cell loss and mossy fiber sprouting. Epilepsia 1993;34:985-995.

5. Parent JM, Yu TW, Leibowitz RT, et al. Dentate granule cell neurogenesis is increased by seizures and contributes to aberrant network reorganization in the adult rat hippocampus. J Neurosci 1997; 17:3727-3738.

6. Ribak CE, Tran PH, Spigelman I, et al. Status epilepticusinduced hilar basal dendrites on rodent granule cells contribute to recurrent excitatory circuitry. J Comp Neurol 2000;428: $240-253$.

7. Scharfman HE, Goodman JH, Sollas AL. Granule-like neurons at the hilar/CA3 border after status epilepticus and their synchrony with area CA3 pyramidal cells: functional implications of seizure-induced neurogenesis. J Neurosci 2000;20: 6144-6158.

8. Dashtipour K, Tran PH, Okazaki MM, et al. Ultrastructural features and synaptic connections of hilar ectopic granule cells in the rat dentate gyrus are different from those of granule cells in the granule cell layer. Brain Res 2001;890:261-271.

9. Jung KH, Chu K, Kim M, et al. Continuous cytosine-b-Darabinofuranoside infusion reduces ectopic granule cells in adult rat hippocampus with attenuation of spontaneous recurrent seizures following pilocarpine-induced status epilepticus. Eur J Neurosci 2004;19:3219-3226.

10. Altman J, Das GD. Autoradiographic and histological evidence of postnatal hippocampal neurogenesis in rats. J Comp Neurol 1965;124:319-335.

11. Altman J. Autoradiographic and histological studies of postnatal neurogenesis. IV. Cell proliferation and migration in the anterior forebrain, with special reference to persisting neurogenesis in the olfactory bulb. J Comp Neurol 1969;137:433-458.

12. Kaplan MS, Hinds JW. Neurogenesis in adult rat: electron microscopic analysis of light radioautographs. Science 1977;197: 1092-1094.

13. Cameron HA, Woolley CS, McEwen BS, Gould E. Differentiation of newly born neurons and glia in the dentate gyrus of the adult rat. Neuroscience 1993;56:337-344.

14. Lois C, Alvarez-Buylla A. Long-distance neuronal migration in the adult mammalian brain. Science 1994;264:1145-1148.

15. Kuhn HG, Dickinson-Anson H, Gage FH. Neurogenesis in the dentate gyrus of the adult rat: age-related decrease of neuronal progenitor proliferation. J Neurosci 1996;16:2027-2033.

16. Bengzon J, Kokaia Z, Elmer E, et al. Apoptosis and proliferation of dentate gyrus neurons after single and intermittent limbic seizures. Proc Natl Acad Sci U S A 1997;94:10432-10437.

17. Parent JM, Valentin VV, Lowenstein DH. Prolonged seizures increase proliferating neuroblasts in the adult rat subventricular zone-olfactory bulb pathway. J Neurosci 2002;22:3174-3188.

18. Parent JM, Janumpalli S, McNamara JO, Lowenstein DH. Increased dentate granule cell neurogenesis following amygdala kindling in the adult rat. Neurosci Lett 1998;247:9-12.

19. Gray WP, Sundstrom LE. Kainic acid increases the proliferation of granule cell progenitors in the dentate gyrus of the adult rat. Brain Res 1998;790:52-59.

20. Scott BW, Wang S, Burnham WM, et al. Kindling-induced neurogenesis in the dentate gyrus of the rat. Neurosci Lett 1998;248:73-76. 
21. Parent JM, Tada E, Fike JR, Lowenstein DH. Inhibition of dentate granule cell neurogenesis with brain irradiation does not prevent seizure-induced mossy fiber synaptic reorganization in the rat. J Neurosci 1999;19:4508-4519.

22. Pleasure SJ, Collins AE, Lowenstein DH. Unique expression patterns of cell fate molecules delineate sequential stages of dentate gyrus development. J Neurosci 2000;20:6095-6105.

23. Elliott RC, Khademi S, Pleasure SJ, et al. Differential regulation of basic helix-loop-helix mRNAs in the dentate gyrus following status epilepticus. Neuroscience 2001;106:79-88.

24. Bagri A, Gurney T, He X, et al. The chemokine SDF1 regulates migration of dentate granule cells. Development 2002; 129:4249-4260.

25. Seki T, Arai Y. Highly polysialylated neural cell adhesion molecule (NCAM-H) is expressed by newly generated granule cells in the dentate gyrus of the adult rat. J Neurosci 1993;13: 2351-2358.

26. Gates MA, Thomas LB, Howard EM, et al. Cell and molecular analysis of the developing and adult mouse subventricular zone of the cerebral hemispheres. J Comp Neurol 1995;361: 249-266.

27. Doetsch F, Alvarez-Buylla A. Network of tangential pathways for neuronal migration in adult mammalian brain. Proc Natl Acad Sci U S A 1996;93:14895-14900.

28. Gleeson JG, Lin PT, Flanagan LA, Walsh CA. Doublecortin is a microtubule-associated protein and is expressed widely by migrating neurons. Neuron 1999;23:257-271.

29. Nacher J, Crespo C, McEwen BS. Doublecortin expression in the adult rat telencephalon. Eur J Neurosci 2001;14:629-644.

30. Nacher J, Rosell DR, McEwen BS. Widespread expression of collapsin response-mediated protein 4 in the telencephalon and other areas of the adult rat central nervous system. J Comp Neurol 2000;424:628-639.

31. Parent JM, Vexler ZS, Gong C, et al. Rat forebrain neurogenesis and striatal neuron replacement after focal stroke. Ann Neurol 2002;52:802-813.

32. Lois C, García-Verdugo JM, Alvarez-Buylla A. Chain migration of neuronal precursors. Science 1996;271:978-981.

33. Peretto P, Merighi A, Fasolo A, Bonfanti L. Glial tubes in the rostral migratory stream of the adult rat. Brain Res Bull 1997; 42:9-21.

34. Seki T, Arai Y. Temporal and spacial relationships between PSA-NCAM-expressing, newly generated granule cells, and radial glia-like cells in the adult dentate gyrus. J Comp Neurol 1999;410:503-513.

35. Thom M, Zhou J, Martinian L, Sisodiya S. Quantitative postmortem study of the hippocampus in chronic epilepsy: seizures do not inevitably cause neuronal loss. Brain 2005;128: $1344-1357$.
36. Amaral DG. A Golgi study of cell types in the hilar region of the hippocampus in the rat. Comp Neurol 1978;182:851-914.

37. Seress L, Pokorny J. Structure of the granular layer of the rat dentate gyrus. A light microscopic and Golgi study. J Anat 1981;133:181-195.

38. Gaarskjaer FB, Laurberg S. Ectopic granule cells of hilus fasciae dentatae projecting to the ipsilateral region inferior of the rat hippocampus. Brain Res 1983;274:11-16.

39. Eriksson PS, Perfilieva E, Bjork-Eriksson T, et al. Neurogenesis in the adult human hippocampus. Nat Med 1998;4: 1313-1317.

40. Gould E, Tanapat P, McEwen BS, et al. Proliferation of granule cell precursors in the dentate gyrus of adult monkeys is diminished by stress. Proc Natl Acad Sci U S A 1998;95: $3168-3171$.

41. Kornack DR, Rakic P. Continuation of neurogenesis in the hippocampus of the adult macaque monkey. Proc Natl Acad Sci U S A 1999;96:5768-5773.

42. Blumcke I, Schewe JC, Normann S, et al. Increase of nestinimmunoreactive neural precursor cells in the dentate gyrus of pediatric patients with early-onset temporal lobe epilepsy. Hippocampus 2001;11:311-321.

43. Mathern GW, Leiphart JL, De Vera A, et al. Seizures decrease postnatal neurogenesis and granule cell development in the human fascia dentata. Epilepsia 2002;43:68-73.

44. Zhao S, Chai X, Forster E, Frotscher M. Reelin is a positional signal for the lamination of dentate granule cells. Development 2004;131:5117-5125.

45. Haas CA, Dudeck O, Kirsch M, et al. Role for Reelin in the development of granule cell dispersion in temporal lobe epilepsy. J Neurosci 2002;22:5797-5802.

46. Ernfors P, Bengzon J, Kokaia Z, et al. Increased levels of messenger RNAs for neurotrophic factors in the brain during kindling epileptogenesis. Neuron 1991;7:165-176.

47. Isackson PJ, Huntsman MM, Murray KD, Gall CM. BDNF mRNA expression is increased in adult rat forebrain after limbic seizures: temporal patterns of induction distinct from NGF. Neuron 1991;6:937-948.

48. Scharfman H, Goodman J, Macleod A, et al. Increased neurogenesis and the ectopic granule cells after intrahippocampal BDNF infusion in adult rats. Exp Neurol 2005;192:348-356.

49. Arvidsson A, Collin T, Kirik D, et al. Neuronal replacement from endogenous precursors in the adult brain after stroke. Nat Med 2002;8:963-970.

50. Nakatomi H, Kuriu T, Okabe $S$, et al. Regeneration of hippocampal pyramidal neurons after ischemic brain injury by recruitment of endogenous neural progenitors. Cell 2002;110: 429-441. 